\title{
On parabolic Whittaker functions II
}

\author{
SERGEY OBLEZIN*
}

\begin{abstract}
We derive a Givental-type stationary phase integral representation for the specified $\mathrm{Gr}_{m, N^{-}}$ Whittaker function introduced in GLO2, which presumably describes the $S^{1} \times U_{N}$-equivariant Gromov-Witten invariants of Grassmann variety $\mathrm{Gr}_{m, N}$. Our main tool is a generalization of Whittaker model for principal series $\mathcal{U}\left(\mathfrak{g l}_{N}\right)$-modules. In particular, our construction includes a representation theory interpretation of the Batyrev-Ciocan-Fontanine-Kim-van Straten toric degeneration of Grassmannian, providing a direct connection between this toric degeneration of $\mathrm{Gr}_{m, N}$ and total positivity for unipotent matrices.
\end{abstract}

\section{Introduction}

Quantum cohomology D-module $Q H^{*}\left(\mathrm{Fl}_{N}\right)$ for complete flag manifold $\mathrm{Fl}_{N}$ of $G L_{N}$ can be identified with the quantum Toda lattice Giv1, GK]. In Giv2 Givental proposed a stationary phase integral formula for the solution (generating function) of $Q H^{*}\left(\mathrm{Fl}_{N}\right)$. Unfortunately, Givental's approach cannot be applied directly to description of quantum cohomology of incomplete (partial) flag variaties, since no relevant Toda lattice associated with a partial flag variaties was known.

Givental's construction possesses a set of remarkable properties, and particularly it involves a degeneration of $\mathrm{Fl}_{N}$ to a certain Gorenstein toric Fano variety. In [BCFKvS1] and BCFKvS2] Batyrev, Ciocan-Fontanine, Kim and van Straten introduced toric degenerations of partial flag manifolds of $G L_{N}$, generalizing Givental's result; they conjectured that the proposed toric varieties describe the quantum cohomology of partial flag manifolds.

In GKLO, GLO1] was given a representation theory proof of Givental's stationary phase integral formula for the complete flag manifold, including a representation theory derivation of Givental's toric degeneration of complete flag manifold in more general context of Lie groups of classical type. In this note we extend the construction of GKLO to Grassmann varieties, giving a representation theory construction of the toric degeneration of Grassmann varieties $\mathrm{Gr}_{m, N}$ proposed by Batyrev, Ciocan-Fontanine, Kim, van Straten in [BCFKvS1] and [BCFKvS2].

The key obstacle in representation theory approach to quantum cohomology of homogeneous spaces until recently was an absence of a relevant Toda lattice associated with partial flag manifolds. Recently such a quantum Toda-type lattice was proposed in GLO2, using a generalization of the classical Whittaker model for principal series representations of $\mathcal{U}\left(\mathfrak{g l}_{N}\right)$. The solution $\Psi_{\lambda}^{(m, N)}\left(x_{1}, \ldots, x_{N}\right)$ to the quantum parabolic Toda lattice was defined in [GLO2] as a certain matrix element in principal series representation, and it was referred to as parabolic Whittaker function, or $\mathrm{Gr}_{m, N^{-}}$Whittaker function. It was conjectured in GLO2] that the specialized parabolic Whittaker function, $\Psi_{\underline{\lambda}}^{(m, N)}(x, 0, \ldots, 0)$, describes the $S^{1} \times U_{N}$-equivariant quantum cohomology of $\mathrm{Gr}_{m, N}$, and

*The work is partially supported by P. Deligne's 2004 Balzan Prize in Mathematics, and by the RFBR 09-0193108-NCNIL-a Grant. 
this conjecture was verified in the case of projective variety $\mathrm{Gr}_{1, N}=\mathbb{P}^{N-1}$. This conjecture is supported by an observation that the specialized symbol $L\left(x, 0, \ldots, 0 ; p_{1}, \ldots, p_{N}\right)$ of the Lax operator associated with the parabolic Toda lattice reproduces the small cohomology algebra $q H^{*}\left(\mathrm{Gr}_{m, N}\right)$ in the form of [AS] and $[\mathrm{K}]$.

In our main result, Theorem 1.1, we propose a stationary phase integral formula for the specialized $\mathrm{Gr}_{m, N}$-Whittaker function $\Psi_{\lambda}^{(m, N)}(x, 0, \ldots, 0)$. Our basic tool is a generalization of the classical Whittaker model for principal series representations of $\mathcal{U}\left(\mathfrak{g l}_{N}\right)$, which extends the construction of generalized Whittaker model from [GLO2]. This result should be compared with the Mellin-Barnes integral representation for $\Psi_{\underline{\lambda}}^{(m, N)}(x, 0, \ldots, 0)$ constructed recently in $\left.\mathrm{O}\right]$. The coincidence of the two integrals in the case $\mathrm{Gr}_{1, N}=\mathbb{P}^{N-1}$ is evident due to a simple transformation of integration variables. For generic $\mathrm{Gr}_{m, N}$ an identification of the two integrals involves a more delicate description of the integration contour of the stationary phase integral than we do in this note; this will be done in a separate note.

Moreover, according to GLO2] the two integral representations for $\Psi_{\underline{\lambda}}^{(m, N)}(x, 0, \ldots, 0)$, the stationary phase one and the Mellin-Barnes one from [O], should be presumably identified with certain correlation functions in the two, type B and type A respectively, mirror dual topological field theories. A detailed analysis of the two integral representations of the $\mathrm{Gr}_{m, N^{-}}$Whittaker function in the framework of GLO2] will be given elsewhere.

Our stationary phase integral representation of $\mathrm{Gr}_{m, N}$-Whittaker function is naturally related to a toric degeneration of the Grassmannian (Corollary 1.2). Namely, we identify the function defining the toric variety 1 (in GLO2 it is given by the superpotential in the mirror dual Landau-Ginzburg model) with phase function $\mathcal{F}_{m, N}$ in our integral formula when the $U_{N}$-equivariance parameters $\underline{\lambda}=\left(\lambda_{1}, \ldots, \lambda_{N}\right)$ are specialized to zero.

Our construction of stationary phase integral uses the Gauss-Givental realization of universal enveloping algebra $\mathcal{U}\left(\mathfrak{g l}_{N}\right)$ in the space of functions on totally positive unipotent (upper-triangular) matrices introduced in GKLO]. It should be emphasized that in our representation theory approach the involved toric degeneration of the (partial) flag manifolds is directly provided by the total positivity property of the Gauss-Givenatal realization of $\mathcal{U}\left(\mathfrak{g l}_{N}\right)$; this connection was established in GLO1] for complete flag manifolds of Lie groups of classical types. One may also refer [GLO1] for a detailed analysis of connections between Gauss-Givental realizations of $\mathcal{U}(\mathfrak{g})$ in general setting and total positivity phenomenon $[\mathrm{L}$.

The paper is organized in the following way. In Section 1 we introduce a generalization of the standard Whittaker model, and define $\mathrm{Gr}_{m, N^{-}}$Whittaker function.In Theorem 1.1 we propose a stationary phase integral representation for $\Psi_{\underline{\lambda}}^{(m, N)}(x, 0, \ldots, 0)$, which extends the Givental's integral formula for complete flag manifold $\mathrm{Fl}_{N}$. Next we describe the arising toric degeneration of $\mathrm{Gr}_{m, N}$ in Corollary 1.2, and identify it with the Batyrev-Ciocan-Fontanine-Kim-van Straten construction BCFKvS1, BCFKvS2]. In Proposition 1.1 we derive the Lax operator for the associated $\mathrm{Gr}_{m, N}$-Toda lattice, and in Proposition 1.2 we find out the first two quantum Hamiltonians of the $\mathrm{Gr}_{m, N^{-}}$-Toda lattice. The second half contains detailed proofs of our main results. In particular, in Section 2 we review on the Gauss-Givental realization of $\mathcal{U}\left(\mathfrak{g l}_{N}\right)$ from GKLO] and construct the $\mathrm{Gr}_{m, N}$-Whittaker vectors; and in Section 3 we verify the integral formula from Theorem 1.1. In Section 4 we prove Propositions 1.1 and 1.2.

Acknowledgments: The author is thankful to A. Gerasimov and D. Lebedev for very useful discussions.

\footnotetext{
${ }^{1}$ In [BCFKvS1] it referred to as "Lax operator of Grassmannian"; one should not mistake this function with the matrix-valued differential operator $\mathcal{L}\left(x, \partial_{x}\right)$ in the present work
} 


\section{The $\mathrm{Gr}_{m, N}$-Whittaker functions}

A Whittaker model for principal series representation of universal enveloping alegbra $\mathcal{U}(\mathfrak{g})$ involves a choice of an $N$-dimensional commutative subalgebra $\mathfrak{a}$ and the Lie algebra decomposition $\mathfrak{g}=$ $\mathfrak{u}_{-} \oplus \mathfrak{a} \oplus \mathfrak{u}_{+}$. Then choosing a pair of characters $\chi_{ \pm}: \mathfrak{u}_{ \pm} \rightarrow \mathbb{C}$ one can reduce the space of functions on $G=$ Lie $(\mathfrak{g})$ to the space of functions on the commutative subgroup Lie $(\mathfrak{a})$. In the case $\mathfrak{g}=\mathfrak{g l}_{N}$ one uses the Gauss triangular decomposition of $\mathfrak{g l}_{N}$ with $\mathfrak{a}$ being the diagonal subalgebra of semisimple elements, and $\mathfrak{u}_{+}$being the nilpotent subalgebras of upper-triangular matrices 2

In GLO2 was proposed a generalization of the standard scheme with $\mathfrak{a} \subset \mathfrak{g l}_{N}$ being a commutative subalgebra, containing both semisimple and nilpotent elements, and similarly for $\mathfrak{u}_{ \pm}$. In the following we use another generalized Whittaker model, using another choice of commutative subalgebra $\mathfrak{a}=\mathfrak{h}^{(m, N)} \subset \mathfrak{g l}_{N}$. In special case of $\mathrm{Gr}_{1, N}=\mathbb{P}^{N-1}$ our choice of the commutative subalgebra $\mathfrak{h}^{(m)} \subset \mathfrak{g l}_{N}$ coincides with the one used in [GLO2] and [0]. For generic $m$ the two commutative subalgebras differ by a simple automorphism, and thus the generalized Whittaker model used in our calculations below is different from GLO2].

Let us consider the triangular decomposition of the Lie algebra $\mathfrak{g l}_{N}$ of real $(N \times N)$-matrices with the standard set of generators $E_{i j}, i, j=1, \ldots, N$. Namely, let $\mathfrak{b}_{ \pm}$be the subalgebras of upper- and lower-triangular matrices, and $\mathfrak{n}_{ \pm}=\left[\mathfrak{b}_{ \pm}, \mathfrak{b}_{ \pm}\right] \subset \mathfrak{b}_{ \pm}$are the radicals of the triangular subalgebras. Then the following holds:

$$
\mathfrak{g l}_{N}=\mathfrak{n}_{-} \oplus \mathfrak{h} \oplus \mathfrak{n}_{+}
$$

Next, let $\Delta=\left\{\alpha_{i} ; i \in I\right\}$ be the set of simple roots indexed by $I=\{1,2, \ldots, N-1\}$, and $R_{+}$ be the set of positive roots. Given an elementary subset $\{m\} \subset I$, let us associate with $m$ the following modification of the triangular decomposition:

$$
\mathfrak{g l}_{N}=\mathfrak{n}_{-}^{(m, N)} \oplus \mathfrak{h}^{(m, N)} \oplus \mathfrak{n}_{+}^{(m, N)},
$$

where the commutative subalgebra $\mathfrak{h}^{(m, N)}$ is spanned by

$$
\begin{array}{cc}
H_{1}=E_{11}+\ldots+E_{m m}, & H_{i}=E_{i, 1}, \quad i=2, \ldots, m \\
H_{j}=E_{j, N}, \quad j=m+1, \ldots, N-1 ; & H_{N}=E_{m+1, m+1}+\ldots+E_{N N} .
\end{array}
$$

The Lie subalgebras $\mathfrak{n}_{ \pm}^{(m)}$ are defined by their set of generators as follows:

$$
\begin{aligned}
\mathfrak{n}_{-}^{(m, N)}=\left\langle E_{m+1,1} ; \quad E_{k i}, i=2, \ldots, m, k=i, \ldots, N ;\right. \\
\left.E_{j+1, j}, j=m+1, \ldots, N-1\right\rangle,
\end{aligned}
$$

and

$$
\begin{aligned}
\mathfrak{n}_{+}^{(m, N)}=\left\langle E_{i-1, i}, i=2, \ldots, m ; \quad\right. & E_{m, N} \\
& \left.E_{k j}, j=m+1, \ldots, N-1, k=1, \ldots, j\right\rangle .
\end{aligned}
$$

One may note that

$$
\operatorname{dim} \mathfrak{h}^{(m, N)}=\operatorname{rank} \mathfrak{g l}_{N}=N, \quad \quad \operatorname{dim} \mathfrak{n}_{ \pm}^{(m, N)}=\operatorname{dim} \mathfrak{n}_{ \pm}=\frac{N(N-1)}{2}
$$

\footnotetext{
${ }^{2}$ Alternatively, one can consider the Iwasawa decomposition $\mathfrak{g l}_{N}=\mathfrak{k} \oplus \mathfrak{a} \oplus \mathfrak{u}_{+}$with $\mathfrak{k} \subset \mathfrak{g l}_{N}$ being the compact subalgebra; then choosing characters of $\mathfrak{k}$ and $\mathfrak{u}_{+}$one can obtain another reduction of the space of functions on $G L_{N}$
} 
Let $H^{(m, N)}$ and $N_{+}^{(m, N)}$ be the Lie groups corresponding to the Lie algebras $\mathfrak{h}^{(m, N)}$ and $\mathfrak{n}_{+}^{(m, N)}$, then an open part $G L_{N}^{\circ}$ (the big Bruhat cell) of $G L_{N}$ allows the following modification of the Gauss decomposition:

$$
G L_{N}^{\circ}=N_{-}^{(m, N)} \cdot H^{(m, N)} \cdot N_{+}^{(m, N)}
$$

A principal series representation $\mathcal{V}_{\underline{\lambda}}$ admits a natural structure of $\mathcal{U}$-module. Let us assume that the action of the commutative subalgebra $\mathfrak{h}^{(m)} \subset \mathfrak{g l}_{N}$ in $\mathcal{V}_{\underline{\lambda}}$ is integrable to the action of commutative subgroup $H^{(m, N)} \subset G L_{N}(\mathbb{R})$. Below we introduce a pair of elements, $\psi_{L}, \psi_{R} \in \mathcal{V}_{\underline{\lambda}}$, generating a pair of Whittaker submodules in $\mathcal{V}_{\underline{\lambda}}, \mathcal{W}_{L}=\mathcal{U} \psi_{L}$ and $\mathcal{W}_{R}=\mathcal{U} \psi_{R}$.

Definition 1.1 The $\mathrm{Gr}_{m, N}$-Whittaker vectors $\psi_{L}$ and $\psi_{R}$ are defined by the following conditions:

$$
\begin{gathered}
\left\{\begin{array}{lc}
E_{m+1,1} \psi_{L}=\hbar^{-1} \psi_{L} ; & \\
E_{k i} \psi_{L}=0, & i=2, \ldots, m, k=i, \ldots, N ; \\
E_{j+1, j} \psi_{L}=\hbar^{-1} \psi_{L}, & j=m+1, \ldots, N-1 ;
\end{array}\right. \\
\left\{\begin{array}{lc}
E_{i-1, i} \psi_{R}=-\hbar^{-1} \psi_{R}, & i=2, \ldots, m ; \\
E_{k j} \psi_{R}=0, & j=m+1, \ldots, N-1, k=1, \ldots, j ; \\
E_{k j} \psi_{L}=(-1)^{\epsilon(m, N)} \hbar^{-1} \psi_{L}, & j=m+1, \ldots, N-1, k=1, \ldots, j ;
\end{array}\right.
\end{gathered}
$$

where $\epsilon(m, N)$ is an integer number and $\hbar$ is an indeterminant.

Lemma 1.1 The introduced $\mathrm{Gr}_{m, N}$-Whittaker vectors define characters of the subalgebras $\mathfrak{n}_{ \pm}^{(m, N)}$ :

$$
\chi_{+}: \mathfrak{n}_{+}^{(m, N)} \longrightarrow \mathbb{C}, \quad \chi_{-}: \mathfrak{n}_{-}^{(m, N)} \longrightarrow \mathbb{C}
$$

Proof: One can readily check that the defining equations (1.6) are compatible with Lie algebra relations in $\mathfrak{n}_{-}^{(m, N)}$; and the same is valid for (1.7) and $\mathfrak{n}_{+}^{(m, N)}$.

Definition 1.2 Given a pair of characters $\chi_{ \pm}$of the opposed nilpotent subalgebras $\mathfrak{n}_{ \pm}^{(m, N)}$, the $\mathrm{Gr}_{m, N}$-Whittaker function associated with the principal series representation $\left(\pi_{\underline{\lambda}}, \mathcal{V}_{\underline{\lambda}}\right)$ is defined as the following matrix element:

$$
\Psi_{\underline{\lambda}}^{(m, N)}(\underline{x})=e^{-x_{1} \frac{m(N-m)}{2}}\left\langle\psi_{L}^{(m, N)}, \pi_{\underline{\lambda}}\left(g\left(x_{1}, \ldots, x_{N}\right)\right) \psi_{R}^{(m, N)}\right\rangle,
$$

where the left and right vectors solve the equations (1.6) and (1.7) respectively, and . Here $g(x)$ is a $H^{(m)}$-valued function given by

$$
g(\underline{x})=\exp \left\{\sum_{i=1}^{N} x_{i} H_{i}\right\},
$$

where $\underline{x}=\left(x_{1}, \ldots, x_{N}\right)$ and the generators $H_{i}, i=1, \ldots, N$ are defined by (1.2).

In the above definition $\langle$,$\rangle denotes a non-degenerate pairing between the Whittaker submodules:$ $\mathcal{W}_{L} \times \mathcal{W}_{R} \rightarrow \mathbb{C}$. 


\subsection{Stationary phase integral and Toric degeneration of $\mathrm{Gr}_{m, N}$}

This part contains our main result; namely, we introduce the stationary phase integral representation for the $\mathrm{Gr}_{m, N}$-Whittaker function, and then establish a direct connection to the toric degeneration of Grassmannian $\mathrm{Gr}_{m, N}$ proposed in [BCFKvS1].

Theorem 1.1 The specialized $\mathrm{Gr}_{m, N}$-Whittaker function (1.9) has the following stationary phase integral representation.

$$
\Psi_{\underline{\lambda}}^{(m, N)}\left(x_{N, 1}, 0, \ldots, 0\right)=\int_{\mathcal{C}} \omega_{m, N} e^{\mathcal{F}_{m, N}(\underline{x})},
$$

where

$$
\begin{gathered}
\mathcal{F}_{m, N}(\underline{x})=\imath\left(\sum_{k=1}^{m} \lambda_{N-m+k}\right) x_{N, 1}+\imath \sum_{n=1}^{N-m}\left(\lambda_{n}-\lambda_{n+1}\right) \sum_{i=1}^{\min (m, n)} x_{n, i} \\
+\imath \sum_{n=1}^{m-1}\left(\lambda_{N-m+n}-\lambda_{N-m+n+1}\right) \sum_{i=n+1}^{\min (N-m+n, m)} x_{N-m+n, i} \\
-\frac{1}{\hbar}\left(e^{-x_{m m}}+e^{x_{N-m, 1}-x_{N, 1}}+\sum_{k=1}^{m} \sum_{i=1}^{N-1-m} e^{x_{i+k-1, k}-x_{i+k, k}}\right. \\
\left.+\sum_{k=1}^{N-m} \sum_{i=1}^{m-1} e^{x_{k+i, i+1}-x_{k+i-1, i}}\right) .
\end{gathered}
$$

and

$$
\omega_{m, N}=\prod_{n=1}^{N-m} \prod_{k=1}^{\min (n, m)} d x_{n, k} \cdot \prod_{n=1}^{m-1} \prod_{i=n+1}^{\min (N-m+n, m)} d x_{N-m+n, i}
$$

The integration contour $\mathcal{C}$ is a slight deformation of $\mathbb{R}^{m(N-m)}$ in $\mathbb{C}^{m(N-m)}$ such that the integrand decreases exponentially.

Proof: The proof is given in Section 3. 
Specifying the parameters $\lambda_{n}=0, n=1, \ldots, N$, the function $\mathcal{F}_{m, N}(\underline{x})$ admits a simple combinatorial structure. Namely, let us consider the following graph:

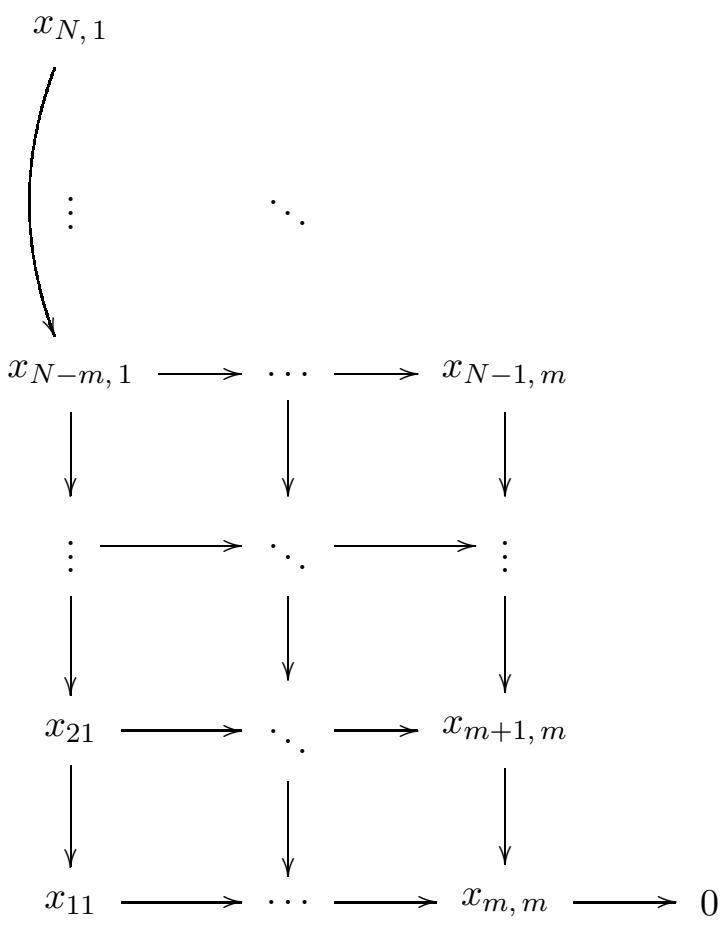

Then let us associate with every arrow $x \rightarrow y$ an exponential function $e^{y-x}$; to any interior vertex $x_{k, i}$ in (1.14) let us assign a pair of exponential functions $a_{k, i}=e^{x_{k-1}-x_{k, i}}$ and $b_{k, i}=e^{x_{k+1, i+1}-x_{k, i}}$. Besides, let $a_{N}=e^{x_{N-m, 1}-x_{N, 1}}$ and $b_{m}=e^{-x_{m m}}$.

Corollary 1.1 The function $\mathcal{F}_{m, N}(\underline{x})(1.12)$ equals to the sum of exponential functions for all the arrows in the graph (1.14):

$$
\mathcal{F}_{m, N}=a_{N}+\sum_{k=2}^{N-m} a_{k, m}+b_{m}+\sum_{i=1}^{m-1} b_{1, i}+\sum_{k=2}^{N-m} \sum_{i=1}^{m-1} a_{k, i}+b_{k, i} .
$$

Actually, the graph (1.14) defines a toric degeneration of the Grassmann variety [BCFKvS1, BCFKvS2]. Namely, the torification of $\mathrm{Gr}_{m, N}$ can be identified with a spectrum of the algebra of functions in $a_{k i}, k=2, \ldots, N-m, i=1, \ldots, m$ and $b_{n j}, n=1, \ldots, N-m, j=1, \ldots, m-1$ modulo the ideal of relations:

$$
\begin{array}{clrl}
a_{k, i} b_{k-1, i}=b_{k, i} a_{k+1, i+1}, & k=2, \ldots, N-m, & i & =1, \ldots, m-1 ; \\
a_{N} b_{m} & \prod_{i=1}^{m-1} a_{N-m, i} \prod_{k=2}^{N-m} b_{k, m} & =q, & q
\end{array}
$$

\subsection{Quantum $\mathrm{Gr}_{m, N}$-Toda lattice}

Actually, the $\mathrm{Gr}_{m, N}$-Whittaker function defines a D-module introduced in [GLO2] and called quantum $\mathrm{Gr}_{m, N}$-Toda lattice. This D-module is provided by the infinitesimal action of the universal enveloping algebra $\mathcal{U}\left(\mathfrak{g l}_{N}\right)$ in our representation $\left(\pi_{\underline{\lambda}}, \mathcal{V}_{\underline{\lambda}}\right)$. In this part we describe the D-module $\mathcal{D}_{m, N}$ defined by (1.9), and then identify $\mathcal{D}_{m, N}$ with the $\mathrm{Gr}_{m, N}$-Toda lattice from [GLO2]. 
The action of the center $\mathcal{Z} \subset \mathcal{U}\left(\mathfrak{g l}_{N}\right)$ of the universal enveloping algebra in principal series representation $\left(\pi_{\underline{\lambda}}, \mathcal{V}_{\underline{\lambda}}\right)$ produces the following action of differential operators, the parabolic Toda lattice Hamiltonians, on $\mathrm{Gr}_{m, N}$-Whittaker function:

$$
\mathcal{H}_{k}^{(m, N)}\left(x, \partial_{x}\right) \cdot \Psi_{\underline{\lambda}}^{(m, N)}(\underline{x})=\hbar^{k} e^{-x_{1} \frac{m(N-m)}{2}}\left\langle\psi_{L}, \pi_{\underline{\lambda}}\left(c_{k} g(\underline{x})\right) \psi_{R}\right\rangle,
$$

for $k=1, \ldots, N$ with $c_{k}, k=1, \ldots, N$ being the Casimir generators of the center $\mathcal{Z}$. The first two Casimir elements are given by:

$$
C_{1}=\sum_{i=1}^{N} E_{i i}, \quad C_{2}=\sum_{\substack{i, j=1 \\ i<j}}^{N}\left(E_{i i} E_{j j}-E_{j i} E_{i j}+\rho_{i} \rho_{j}\right)-\sum_{i=1}^{N} \rho_{i} E_{i i},
$$

where $\rho_{i}=(N+1-2 i) / 2, i=1, \ldots, N$.

Proposition 1.1 Action of the first two Casimir generators (1.17) have the following explicit form:

$$
\begin{gathered}
\mathcal{H}_{1}^{(m, N)}=\hbar \frac{\partial}{\partial x_{1}}+\hbar \frac{\partial}{\partial x_{N}} \\
\mathcal{H}_{2}^{(m, N)}=\hbar^{2}\left\{\frac{\partial^{2}}{\partial x_{1} \partial x_{N}}+\sum_{1 \leq i \leq j \leq m}\left(\left(-x_{i}\right)^{1-\delta_{i, 1}} \frac{\partial}{\partial x_{i}}\right)\left(x_{j} \frac{\partial}{\partial x_{j}}\right)\right. \\
+\sum_{m+1 \leq i \leq j \leq N}\left(x_{i} \frac{\partial}{\partial x_{i}}\right)\left(x_{j}^{1-\delta_{j, N}} \frac{\partial}{\partial x_{j}}\right)-\sum_{k=1}^{m}(k-1) x_{k} \frac{\partial}{\partial x_{k}} \\
\left.-\sum_{k=m+1}^{N}(N+1-k) x_{k} \frac{\partial}{\partial x_{k}}\right\}-\hbar\left\{\sum_{i=1}^{m-1}\left(-x_{i}\right)^{1-\delta_{i, 1}} \frac{\partial}{\partial x_{i+1}}+\sum_{j=m+1}^{N-1} x_{j+1}^{1-\delta_{j, N-1}} \frac{\partial}{\partial x_{j}}\right\} \\
+(-1)^{\delta_{m, N-1}+\epsilon(m, N)} x_{m}^{1-\delta_{m, 1}} x_{m+1}^{1-\delta_{m, N-1}} e^{x_{N}-x_{1}}-\frac{\hbar^{2}}{24}(N-1)(N-2)(N-3) .
\end{gathered}
$$

Proof: The first statement is trivial. The proof of the second formula is given in Section 4 .

Remark 1.1 The Hamiltonians (1.18) coincide (up to signs in accordance with the choice of signs in (1.10) ) with the first two $\mathrm{Gr}_{m, N}$-Toda Hamiltonians from [GLO2]. Let us emphasize that although for generic $m$ the $\mathrm{Gr}_{m, N}$-Whittaker vectors (1.6) and (1.7) are different from the ones introduced in [GLO2], the two (generalized) Whittaker models: the one from [GLO2], and its modification introduced above, produce the same $\mathrm{Gr}_{m, N}$-Toda D-module. In particular, the Hamiltonians (1.16) are identical to the parabolic Toda Hamiltonians from [GLO2], and after specifying $x_{2}=\ldots=$ $x_{N}=0$ the symbols of Hamiltonians $\mathcal{H}_{k}^{(m, N)}, k=1, \ldots, N$ generate the small quantum cohomology algebra $q H^{*}\left(\mathrm{Gr}_{m, N}\right)$.

Let $\mathcal{D}_{m, N}$ be the D-module generated by the Hamiltonians $\mathcal{H}_{k}^{(m, N)}, k=1, \ldots, N$ as a module over the algebra of differential operators $\mathcal{D}\left(e^{x}, \partial_{x}, \hbar\right)$ :

$$
\mathcal{D}_{m, N} \simeq \mathcal{D}\left(e^{x}, \partial_{x}, \hbar\right) / \mathcal{T}_{m, N}, \quad \mathcal{T}_{m, N}=\left\langle\mathcal{H}_{1}^{(m, N)}\left(x, \partial_{x}\right), \ldots, \mathcal{H}_{N}^{(m, N)}\left(x, \partial_{x}\right)\right\rangle .
$$

Equivalently, the D-module $\mathcal{D}_{m, N}$ can be defined in terms of the quantum Lax operator, the following $\operatorname{Mat}(N, \mathbb{R})$-valued differential operator:

$$
\mathcal{L}\left(\underline{x}, \partial_{\underline{x}}\right) \cdot \Psi_{\underline{\lambda}}^{(m, N)}(\underline{x}):=\hbar \sum_{i, j=1}^{N} e_{i j} e^{-x_{1} \frac{m(N-m)}{2}}\left\langle\psi_{L}, \pi_{\underline{\lambda}}\left(E_{i j} g(\underline{x})\right) \psi_{R}\right\rangle,
$$

where $\left(e_{i j}\right)_{k n}=\delta_{i k} \delta_{j n}$, for $i, j, k, n=1, \ldots, N$ are the matrix units. 
Proposition 1.2 The quantum Lax operator $\mathcal{L}\left(x_{1}, \ldots, x_{N} ; \partial_{x_{1}}, \ldots, \partial_{x_{N}}\right)=\left\|\mathcal{L}_{i j}\right\|, i, j=1, \ldots, N$ has the following form:

$$
\begin{gathered}
\mathcal{L}_{k, 1}=\hbar \partial_{x_{k}}, \quad k=1, \ldots, m ; \quad \mathcal{L}_{m+1,1}=-1, \\
\mathcal{L}_{k, 1}=0, \quad k=m+2, \ldots, N ; \quad \mathcal{L}_{k, j}=0, \quad j=2, \ldots, m, k=j, \ldots, N ; \\
\mathcal{L}_{a+1, a}=-1, \quad \mathcal{L}_{k, a}=0, \quad k=a+2, \ldots, N, a=m+1, \ldots, N-1 ; \\
\mathcal{L}_{1, k}=-\delta_{k, 2}+\left(1-\delta_{k, m}\right) x_{k+1}+x_{k} \partial_{x_{1}}+\sum_{n=2}^{m} x_{k} x_{n} \partial_{x_{n}}, \quad k=2, \ldots, m ; \\
\mathcal{L}_{k, i}=\delta_{i, k+1}+\hbar x_{i} \partial_{x_{k}}, \quad k=2, \ldots, m-1, \quad i=k+1, \ldots, m ; \\
\mathcal{L}_{1, a}=-(-1)^{\epsilon(m, N)} x_{a} x_{m} e^{x_{N}-x_{1}}, \quad \mathcal{L}_{k, a}=0, \quad k=2, \ldots, m-1 \\
\mathcal{L}_{m, a}=(-1)^{\epsilon(m, N)} x_{a} e^{x_{N}-x_{1}}, \quad a=m+1, \ldots, N-1 ; \\
\mathcal{L}_{m, N}=-(-1)^{\epsilon(m, N)} e^{x_{N}-x_{1}} ; \\
\mathcal{L}_{a, N}=\hbar \partial_{x_{a}}, a=m+1, \ldots, N-1 ; \\
\mathcal{L}_{a a}=\hbar x_{a} \partial_{x_{a}}, \quad \sum_{n-1}^{N-1} x_{a} \partial_{x_{a}} .
\end{gathered}
$$

Proof: The proof is given in Section 4.

The symbol of the quantum Lax operator is referred to as the Lax matrix $L\left(x_{1}, \ldots, x_{N} ; p_{1}, \ldots, p_{N}\right)$.

Corollary 1.2 (i) The Lax matrix $L(\underline{x} ; \underline{p})$ of (1.20) and the Lax matrix introduced in [GLOQ] have identical characteristic polynomials.

(ii) The specialized Lax matrix $L\left(x_{1}, 0, \ldots, 0 ; p_{1}, \ldots, p_{N}\right)=\left\|L_{i j}\right\|, i, j=1, \ldots, N$ is given by

$$
\begin{gathered}
L_{k, 1}=p_{k}, \quad k=1, \ldots, m ; \quad L_{m+1,1}=-1, \\
L_{k, 1}=0, \quad k=m+2, \ldots, N ; \quad L_{k, j}=0, \quad j=2, \ldots, m, k=j, \ldots, N ; \\
L_{a+1, a}=-1, \quad L_{a a}=L_{k, a}=0, \quad k=a+2, \ldots, N, \quad a=m+1, \ldots, N-1 ; \\
L_{i, i+1}=-1, \quad i=1, \ldots, m-1 ; \\
L_{a, N}=-p_{a}, \quad a=m+1, \ldots, N-1 ; \quad L_{m, N}=-(-1)^{\epsilon(m, N)} e^{-x_{1}}
\end{gathered}
$$

It defines the small quantum cohomology algebra $q H^{*}\left(\mathrm{Gr}_{m, N}\right)$ in the form of $[A S],[K]$.

Proof: One can readily check that the matrix $\left\|L_{i j}\right\|$ and matrix $A$ in [AS] defining $q H^{*}\left(\mathrm{Gr}_{m, N}\right)$ have identical characteristic polynomials, and thus $\operatorname{det}\left(\lambda+\left\|L_{i j}\right\|\right)$ is the generating function of the ideal for the small quantum cohomology algebra.

\section{Gelfand-Zetlin graphs, paths, and $\mathrm{Gr}_{m, N^{-}}$-Whittaker vectors}

In this section we recall the Gauss-Givental realization of the universal enveloping algebra $\mathcal{U}=$ $\mathcal{U}\left(\mathfrak{g l}_{N}\right)$ introduced in [GKLO]. In the second part of this Section we apply this construction to derivation of the $\mathrm{Gr}_{m, N^{-}}$Whittaker vectors, solving the defining relations (1.6) and (1.7). 
Actually, the construction of Gauss-Givental realization of principal series $\mathcal{U}$-modules originates from the total positivity phenomenon in unipotent varieties developed by Lusztig [L]; a detailed study of connections between Gauss-Givental realizations of $\mathcal{U}(\mathfrak{g})$ and total positivity can be found in GLO1].

2.0.1 Let $\mathcal{M}_{N}$ be the space of meromorphic functions in $e^{x_{n, k}}, n=1, \ldots, N-1 ; k=1, \ldots, n$, then the standard generators $E_{i j}, i, j=1, \ldots, N$ of $\mathfrak{g l}_{N}$ admit the following realization by first-order differential operators in $\mathcal{M}_{N}$ :

$$
\begin{aligned}
E_{i, i} & =\mu_{i}-\sum_{k=1}^{i-1} \frac{\partial}{\partial x_{N+k-i, k}}+\sum_{k=i}^{N-1} \frac{\partial}{\partial x_{k, i}} \\
E_{i, i+1} & =-\sum_{n=1}^{i} e^{x_{N-1-i+n, n}-x_{N-i+n, n}} \sum_{k=1}^{n}\left\{\frac{\partial}{\partial x_{N-1-i+k, k}}-\frac{\partial}{\partial x_{N-1-i+k, k-1}}\right\}, \\
E_{i+1, i} & =\sum_{n=1}^{N-i} e^{x_{n+i, i+1}-x_{k+i-1, i}}\left[\mu_{i}-\mu_{i+1}+\sum_{k=1}^{n}\left\{\frac{\partial}{\partial x_{i+k-1, i}}-\frac{\partial}{\partial x_{i+k-1, i+1}}\right\}\right],
\end{aligned}
$$

where $x_{N, i}=0, i=1, \ldots, N$ is assumed.

The universal enveloping algebra acts in $\mathcal{V}_{\underline{\lambda}} \subset \mathcal{M}_{N}$ by differential operators (2.1) with

$$
\mu_{n}=\imath \lambda_{n}-\rho_{n}^{(N)}, \quad \rho_{n}=n-\frac{N+1}{2}, \quad n=1, \ldots, N
$$

and the Whittaker submodules $\mathcal{W}_{L, R} \subset \mathcal{V}_{\underline{\lambda}} \subset \mathcal{M}_{N}$ are spanned by $\prod_{1 \leq k \leq i \leq N-1} e^{n_{k, i} x_{k, i}} \psi_{L, R}$ with $n_{k, i} \in \mathbb{Z}$. The non-degenerate pairing between the Whittaker modules is given by

$$
\left\langle\phi_{1}, \phi_{2}\right\rangle=\int_{\mathcal{C}} \mu_{N}(x) \overline{\phi_{1}} \phi_{2}, \quad \phi_{1} \in \mathcal{W}_{L}, \quad \phi_{2} \in \mathcal{W}_{R}
$$

where the integration contour is a slight deformation of $\mathbb{R}^{N(N-1) / 2}$ in $\mathbb{C}^{N(N-1) / 2}$ such that the integrand exponentially decreases for $\phi_{1}=\psi_{L}$ and $\phi_{2}=\psi_{R}$, and the measure $\mu_{N}(x)$ is given by

$$
\mu_{N}(x)=\prod_{k=1}^{N-1} \prod_{i=1}^{k} e^{-x_{k, i}} d x_{k, i}
$$

One can readily check that thus defined pairing (2.3) between $\mathcal{W}_{L}$ and $\mathcal{W}_{R}$ possesses the following property:

$$
\left\langle X \cdot \phi_{1}, \phi_{2}\right\rangle=-\left\langle\phi_{1}, X \cdot \psi_{2}\right\rangle, \quad X \in \mathfrak{g l}_{N}, \quad \phi_{1} \in \mathcal{W}_{L}, \quad \phi_{2} \in \mathcal{W}_{R}
$$

2.0.2 The Gauss-Givental realization of $\mathcal{U}\left(\mathfrak{g l}_{N}\right)$ possesses a distinguished combinatorial structure 
arising from the Gelfand-Zetlin graph (see [GLO1]):

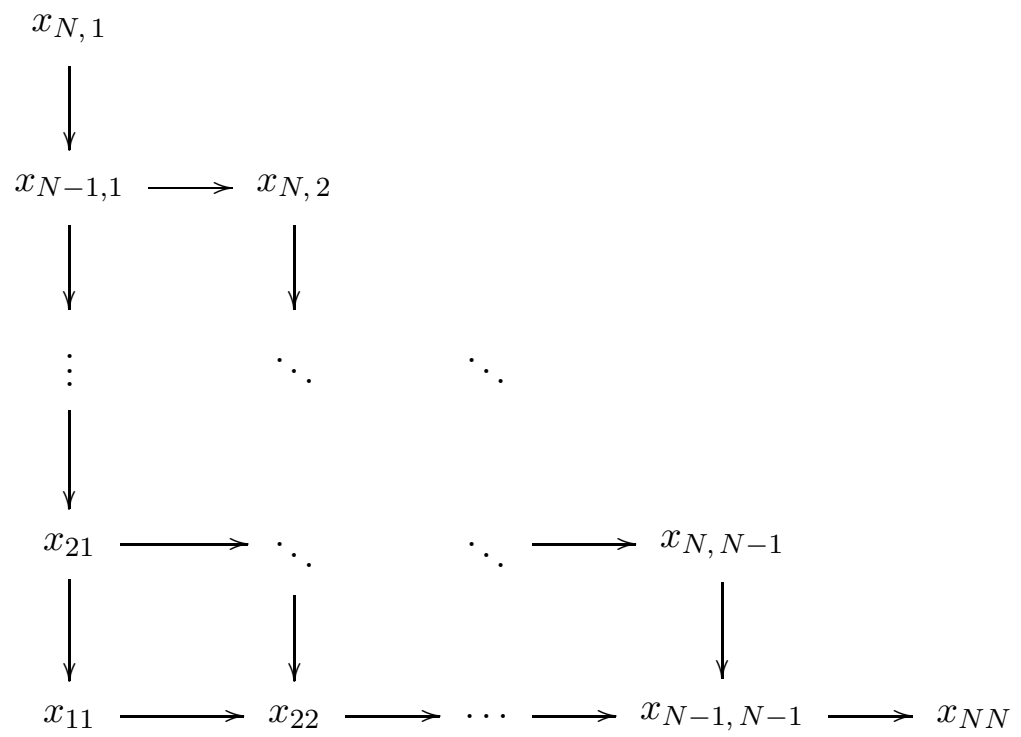

Namely, let $\mathcal{I}_{N}$ be the set of vertices in the Gelfand-Zetlin graph:

$$
\mathcal{I}_{N}=\left\{(n, j) \in \mathbb{Z}_{+}^{2} ; 1 \leq j \leq n \leq N\right\}
$$

there is a tautological embedding $\mathcal{I}_{m} \subset \mathcal{I}_{N}$, for any $0<m<N$. Given $(n, j) \in \mathcal{I}_{N-1} \subset \mathcal{I}_{N}$ let $A_{n, j}^{r}$ be the following function attached to a vertex $x_{n, i}$ in (2.6):

$$
A_{n, j}^{r}=\sum_{I_{r}} \prod_{\alpha=1}^{r} e^{x_{n+i_{\alpha}-\alpha, j+i_{\alpha}}-x_{n+i_{\alpha}-\alpha-1, j+i_{\alpha}}}
$$

where the summation goes over the strict partitions

$$
I_{r}=\left(i_{1}<\ldots<i_{r}\right) \in \mathbb{Z}_{+}^{r}, \quad i_{\alpha} \leq N-n+\alpha, \quad \alpha=1, \ldots, r .
$$

The function $A_{n, j}^{r}(k)$ satisfy the following evident relation:

$$
A_{n, j}^{r}=A_{n+1, j+1}^{r}+A_{n, j+1}^{r-1} e^{x_{n, j+1}-x_{n+1, j+1}} .
$$

Also for $(n, j) \in \mathcal{I}_{N-1} \subset \mathcal{I}_{N}$ let us introduce the function $B_{n, i}$ given by

$$
B_{n, j}(k)=\sum_{I_{k+j-n-1}^{*}} e^{x_{n, j}-x_{n+1, j}} \prod_{\alpha=1}^{k+j-n-1} e^{x_{n+i_{\alpha}+\alpha, j+\imath_{\alpha}}-x_{n+i_{\alpha}-\alpha+1, j+i_{\alpha}}},
$$

where the summation goes over the partitions

$$
I_{k+j-n-1}^{*}=\left(i_{1} \leq \ldots \leq i_{k+j-n-1}\right) \in \mathbb{Z}_{+}^{k+j-n-1} .
$$

The function $B_{n, j}$ satisfies the following relation:

$$
B_{n, j}(k) e^{x_{n+1, j}-x_{n, j}}=B_{n+1, j}(k)+B_{n+2, j+1}(k) .
$$

Actually, the relations (2.8) and (2.10) are direct consequence of the "box relations" (1.15). 
2.0.3 With respect to an obvious symmetry of the graph (1.14), let us introduce the following pair of functions:

$$
{ }^{\top} A_{n, j}^{r}=\sum_{I_{r-1}^{*}} e^{x_{n+1, j+1}-x_{n, j}} \prod_{\alpha=1}^{r-1} e^{x_{n+i_{\alpha}+\alpha+1, j+\alpha+1}-x_{n+i_{\alpha} \alpha, j+\alpha}},
$$

where the summation goes over partitions

$$
I_{r-1}^{*}=\left(i_{1} \leq \ldots \leq i_{r-1}\right) \in \mathbb{Z}_{\geq 0}^{r-1}, \quad i_{\alpha} \leq N-n-r, \quad \alpha=1, \ldots, r-1 ;
$$

and

$$
{ }^{\top} B_{n, j}(k)=\sum_{I_{j-k}} \prod_{\alpha=1}^{j-k} e^{x_{n+i_{\alpha}-\alpha+1, j+1-\alpha}-x_{n+i_{\alpha}-\alpha, j-\alpha}},
$$

where the summation goes over strict partitions

$$
I_{j-k}=\left(i_{1}<\ldots<i_{j-k}\right) \in \mathbb{Z}_{+}^{j-k}, \quad i_{\alpha} \leq N-n+\alpha, \quad \alpha=1, \ldots, j-1 .
$$

Analogously to the the functions $A_{n, j}^{r}$ and $B_{n, j}$, the "box relations" (1.15) imply the following relations for the introduced functions (2.11) and (2.12):

$$
{ }^{\top} A_{n, j}^{r} e^{x_{n+1, j+1}-x_{n, j}}={ }^{\top} A_{n+1, j+1}^{r-1}+{ }^{\top} A_{n+2, j+1}^{r-1} ;
$$

and

$$
{ }^{\top} B_{n, j}={ }^{\top} B_{n+1, j}+{ }^{\top} B_{n, j-1} e^{x_{n+1, j}-x_{n, j-1}} .
$$

2.0.4 In fact, the summations in (2.7), (2.13), and (2.9), (2.14) can be readily interpreted as sums over paths in Gelfand-Zetlin graph. More precisely, the functions

$$
P_{n, j}^{r}(k):=A_{n, j}^{r+n-k-j} \cdot B_{n, j}(k), \quad \quad{ }^{\top} P_{n, j}^{r}:={ }^{\top} A_{n, j}^{r+k-j} \cdot{ }^{\top} B_{n, j},
$$

are represent sums over all paths (with certain restrictions) of length $r$ passing through a vertex $x_{n, j}$ on the Gelfand-Zetlin graph; the paths from $P_{n, j}^{r}(k)$ are starting at horizontal line $\left\{x_{a, b} ; a-b=k\right\}$, and the paths from ${ }^{\top} P_{n, j}^{r}(k)$ are starting at vertical line $\left\{x_{a, b} ; b=k\right\}$ in graph (2.6).

Moreover, the generators $E_{i j}$ of Lie algebra $\mathfrak{g l}_{N}$ in Gauss-Givental realization (2.1) admit a distinct description in terms of certain paths in graph (2.6). Namely, for any vertex $x_{n, j}$ let us introduce the following pair of differential operators:

$$
\begin{aligned}
D_{n, j} & =\frac{\partial}{\partial x_{n+1-j, 1}}+\sum_{i=1}^{j-1}\left(\frac{\partial}{\partial x_{n+1+i-j, i+1}}-\frac{\partial}{\partial x_{n+1+i-j, i}}\right), \\
{ }^{\top} D_{n, j}^{\mu} & =\mu_{j}-\mu_{j+1}+\frac{\partial}{\partial x_{j, j}}+\sum_{i=1}^{n-j}\left(\frac{\partial}{\partial x_{i+j, j}}-\frac{\partial}{\partial x_{i+j, j+1}}\right) .
\end{aligned}
$$

Proposition 2.1 The Lie algebra generators $E_{i j}$ have the following combinatorial realization in terms of the Gelfand-Zetlin graph (2.6):

$$
\begin{aligned}
E_{n, j} & =\sum_{k=j}^{n-1}(-1)^{k+1} \sum_{i=0}^{N-n}{ }^{\top} P_{k+i, k}^{n-j}(j)^{\top} D_{k+i, k}^{\mu}, \quad n>j \\
E_{n, i} & =\sum_{k=1}^{i-n}(-1)^{k} \sum_{j=0}^{n-1} P_{N+k-i+j, j+1}^{i-n}(N-n) D_{N-i+k+j, j+1},
\end{aligned}
$$

for $n<i$. 
Corollary 2.1 The elements $E_{m, 1}$ and $E_{m, N}$ have the following differential operators in the GaussGivental realization (2.1).

$$
\begin{aligned}
& E_{n, N}=\sum_{k=1}^{N+1-n}(-1)^{k+1} \sum_{i=1}^{n} P_{k+i-1, i}^{n+1-i}\left\{\frac{\partial}{\partial x_{k+i-1,1}}+\sum_{j=2}^{i}\left(\frac{\partial}{\partial x_{k+i-2, i}}-\frac{\partial}{\partial x_{k+i-2, i}}\right)\right\} ; \\
& E_{n, 1}=\sum_{k=1}^{n-1}(-1)^{k} \sum_{i=1}^{N-n} P_{k+i, i}^{n-1}\left\{\mu_{k}-\mu_{k+1}+\frac{\partial}{\partial x_{k k}}+\sum_{j=1}^{i}\left(\frac{\partial}{\partial x_{k+j, k}}-\frac{\partial}{\partial x_{k+j, k+1}}\right)\right\},
\end{aligned}
$$

where $P_{n, j}^{r}:=P_{n, j}^{r}(1)$, and ${ }^{\top} P^{r}:={ }^{\top} P_{n, j}^{r}(N-1)$.

Proof: Direct calculations of commutators, using (2.1).

\subsection{Derivation of $\mathrm{Gr}_{m, N^{-}}$Whittaker vectors}

In this part we solve the defining equations (1.6) and (1.7) and find out the $\mathrm{Gr}_{m, N^{-}}$-Whittaker vectors.

Proposition 2.2 The following $\mathrm{Gr}_{m, N}$-Whittaker vectors satisfy the defining equations (1.6) and (1.7) in realization (2.1):

$$
\begin{aligned}
\psi_{L}^{(m, N)}=\frac{1}{C_{m, N}^{L}} \exp \{ & -\sum_{n=1}^{N-1}\left(\mu_{n}-\mu_{n+1}\right) \sum_{i=1}^{n} x_{n, i}+\sum_{k=1}^{m-1} \mu_{N-1-k} x_{N-1-k, 1} \\
-\frac{1}{\hbar}\left(e^{x_{N-m, 1}}+\right. & \sum_{k=1}^{m} \sum_{i=1}^{N-m-1} e^{x_{i+k-1, k}-x_{i+k, k}}+\sum_{k=m+1}^{N-1}\left[e^{x_{N-1, k}}\right. \\
& \left.\left.\left.+\sum_{i=1}^{N-k-1} e^{x_{i+k-1, k}-x_{i+k, k}}\right]\right)\right\}
\end{aligned}
$$

and

$$
\begin{aligned}
& \psi_{R}^{(m, N)}=\frac{1}{C_{m, N}^{R}} \exp \left\{-\sum_{k=m+1}^{N-1} \mu_{k} x_{k k}-\frac{1}{\hbar}\left(e^{-x_{m m}}+\sum_{k=1}^{N-m} \sum_{i=1}^{m-1} e^{x_{k+i, i+1}-x_{k+i-1, i}}\right.\right. \\
&\left.\left.+\sum_{k=1}^{m-1}\left[e^{-x_{N-1, k}}+\sum_{i=1}^{k-1} e^{x_{N-k+i, i+1}-x_{N-k+i-1, i}}\right]\right)\right\}
\end{aligned}
$$

where

$$
C_{m, N}^{L}=\prod_{\substack{i, j=N-m+1 \\ i<j}}^{N} \hbar^{\rho_{i}-\imath \lambda_{j}} \Gamma\left(-\imath \lambda_{j}-\rho_{i}\right), \quad C_{m, N}^{R}=\prod_{\substack{i, j=m+1 \\ i<j}}^{N} \hbar^{\imath \lambda_{j}-\rho_{i}} \Gamma\left(\rho_{i}-\imath \lambda_{j}\right) .
$$

Proof. Our proof of Proposition 2.1 is based on an verification of the defining equations (1.6), (1.7), using the Gauss-Givental realization (2.1).

Actually, the expressions (2.19) and (2.20) (with specialized parameters $\mu_{n}=0, n=1, \ldots, N$ ) have definite interpretation in terms of arrows in (2.6) defined by the equations (1.6), (1.7), respectively. In this way the graph (1.14) is a subgraph of (2.6), built of the corresponding arrows from (2.19) and (2.20). 
2.1.1 At first let us observe that the action of the Cartan generators $E_{i i}, i=2, \ldots, N-1$ fixes a dependence of the Whittaker vectors on the parameters $\left.\mu_{1}, \ldots, \mu_{N}\right)$. Namely, the following holds:

$$
E_{k k} \cdot \exp \left\{-\sum_{n=1}^{N-1}\left(\mu_{n}-\mu_{n+1}\right) \sum_{i=1}^{n} x_{n, i}+\sum_{k=1}^{m-1} \mu_{N-1-k} x_{N-1-k, 1}\right\}=0
$$

for $k=2, \ldots, m$, and

$$
E_{a a} \cdot e^{-\left(\mu_{m+1} x_{m+1, m+1}+\ldots+\mu_{N-1} x_{N-1, N-1}\right)}=0, \quad a=m+1, \ldots, N-1 .
$$

2.1.2 Besides, the action of differential operators $E_{i i}$ have the following properties:

$$
E_{k k} \cdot e^{x_{n, k}-x_{n+1, k}}=0, \quad k=2, \ldots, m
$$

when $n=2, \ldots, N-m, k=2, \ldots, m$, and

$$
E_{a a} \cdot e^{x_{n+1, k+1}-x_{n, k}}=0, \quad a=m+1, \ldots, N-1,
$$

when $n=2, \ldots, N+1-m, k=1, \ldots, m-1$. Also, taking into account that $E_{k k}, k=2, \ldots, m$ annihilate any function in $x_{n, 1}, n=1, \ldots, N-m$, and $E_{a a}, m+1, \ldots, N-1$ annihilate any function in $x_{n n}, n=1, \ldots, m$, one can deduce that (2.19) and (2.20) satisfy

$$
E_{k k} \psi_{L}^{(m, N)}=0, \quad k=2, \ldots, m ; \quad E_{a a} \psi_{R}^{(m, N)}=0, \quad a=m+1, \ldots, N-1 .
$$

2.1.3 Next for $\psi_{R}^{(m, N)}$ one finds out the following:

$$
E_{k-1, k} \psi_{R}^{(m, N)}=-\frac{1}{\hbar} \psi_{R}^{(m, N)}, \quad k=2, \ldots, m
$$

since

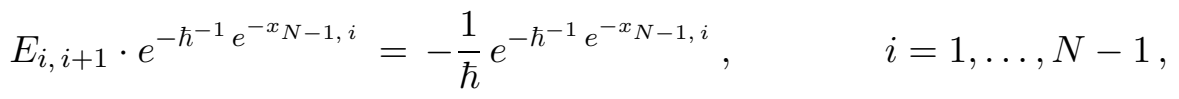

and

$$
E_{i, i+1} \cdot \exp \left\{-\frac{1}{\hbar} \sum_{j=1}^{m-1} e^{x_{N+j-i, j+1}-x_{N+j-i-1, j}}+e^{x_{N+j-i+1, j+1}-x_{N+j-i, j}}\right\}=0, \quad i=1, \ldots, N-1
$$

for any $m=1, \ldots, N-1$, is due to the "box relations" (1.15,1):

$$
e^{x_{N+j-i-1, j}-x_{N+j-i, j}} \cdot e^{x_{N+j-i, j+1}-x_{N+j-i-1, j}}=e^{x_{N+j-i+1, j+1}-x_{N+j-i, j}} \cdot e^{x_{N+j-i, j+1}-x_{N+j-i+1, j+1}},
$$

where $e^{x_{N+j-i-1, j}-x_{N+j-i, j}}$ with $e^{x_{N+j-i, j+1}-x_{N+j-i+1, j+1}}$ come from coefficients in $E_{i, i+1}$, and $e^{x_{N+j-i, j+1}-x_{N+j-i-1, j}}$ with $e^{x_{N+j-i+1, j+1}-x_{N+j-i, j}}$ are from the exponent in (2.20).

2.1.4 Similarly for the left $(m, N)$-Whittaker vectors one obtains:

$$
E_{a+1, a} \psi_{L}^{(m, N)}=-\frac{1}{\hbar} \psi_{L}^{(m, N)}, \quad a=m+1, \ldots, N-1
$$

since for $E_{j+1, j}^{0}$ being the generators (2.1) with specialized parameters $\mu_{j}=\mu_{j+1}=0$ :

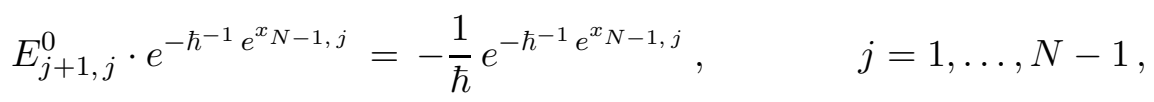


and

$$
E_{j+1, j}^{0} \cdot \exp \left\{-\frac{1}{\hbar} \sum_{i=1}^{r-1} e^{x_{j+i, j}-x_{j+i-1, j}}+e^{x_{j+1+i, j+1}-x_{j+i, j+1}}\right\}=0, \quad j=1, N-1,
$$

for any $r=2, \ldots, N-1$ holds due to the "box relations" (1.15,1):

$$
e^{x_{j+i-1, j}-x_{j+i, j}} \cdot e^{x_{j+i, j+1}-x_{j+i-1, j}}=e^{x_{j+1+i, j+1}-x_{j+i, j}} \cdot e^{x_{j+1+i, j+1}-x_{j+i, j+1}},
$$

where $e^{x_{j+i, j+1}-x_{j+i-1, j}}$ with $e^{x_{j+1+i, j+1}-x_{j+i, j}}$ come from coefficients of $E_{j+1, j}$, and $e^{x_{j+i-1, j}-x_{j+i, j}}$ with $e^{x_{j+1+i, j+1}-x_{j+i, j+1}}$ are from the exponent of (2.19).

2.1.5 At last to verify the remaining defining relations

$$
E_{m, N} \psi_{R}^{m, N}=-\hbar^{-1} \psi_{R}^{m, N}, \quad E_{m+1,1} \psi_{L}^{m, N}=-\hbar^{-1} \psi_{L}^{m, N},
$$

for the $(m, N)$-Whittaker vectors, we use the explicit form (2.18) of the generators $E_{m, N}$ and $E_{m+1,1}$ and apply the relations (2.8), (2.13), and (2.10), (2.14).

\section{Proof of Theorem 1.1}

In this part we apply the results of previous Section to derive the integral formula (1.11) for $\Psi_{\underline{\lambda}}^{(m, N)}(x, 0 \ldots, 0)$. Namely, into (1.9) using (2.3) one readily obtains:

$$
\begin{aligned}
& \Psi_{\underline{\lambda}}^{(m, N)}(x, 0 \ldots, 0)=e^{-x \frac{m(N-m)}{2}}\left\langle\psi_{L}^{(m, N)}, e^{x\left(E_{11}+\ldots+E_{m m}\right)} \psi_{R}^{(m, N)}\right\rangle \\
= & e^{-x \frac{m(N-m)}{2}} \int_{\mathcal{C}} \prod_{n=1}^{N-1} \prod_{i=1}^{n} e^{-x_{n, i}} d x_{n, i} \overline{\psi_{L}^{m, N}(\underline{x})} e^{x\left(E_{11}+\ldots+E_{m m}\right)} \psi_{R}^{(m, N)}(\underline{x}) .
\end{aligned}
$$

Then one picks from (2.1):

$$
E_{11}+\ldots+E_{m m}=\sum_{k=1}^{m} \mu_{k}+\sum_{n=1}^{N-m} \sum_{i=1}^{\min (m, n)} \frac{\partial}{\partial x_{n, i}}+\sum_{n=1}^{m-1} \sum_{i=n+1}^{\min (N-m+n, m)} \frac{\partial}{\partial x_{N-m+n, i}}
$$

and substituting this together with (2.19) and (2.20) into (3.1) one finds out the following:

$$
\begin{gathered}
\ldots=\frac{1}{\overline{C_{m, N}^{L}} C_{m, N}^{R}} \int_{\mathcal{C}} \prod_{n=1}^{N-1} \prod_{i=1}^{n} d x_{n i} e^{\sum_{k=1}^{m-1} \overline{\mu_{N-1-k}} x_{N-1-k, 1}-\sum_{k=m+1}^{N-1} \mu_{k} x_{k k}} \\
\times \exp \left\{\imath \sum_{n=1}^{N-1}\left(\lambda_{n}-\lambda_{n+1}\right) \sum_{i=1}^{n} x_{n, i}+\imath \sum_{k=1}^{m} \lambda_{N-m+k} x-\frac{1}{\hbar}\left(e^{x_{N-m, 1}-x}\right.\right. \\
+\sum_{k=1}^{m} \sum_{i=1}^{N-m-1} e^{x_{i+k-1, k}-x_{i+k, k}}+\sum_{k=m+1}^{N-1}\left[e^{x_{N-1, k}}+\sum_{i=1}^{N-k-1} e^{x_{i+k-1, k}-x_{i+k, k}}\right] \\
+e^{-x_{m m}}+\sum_{k=1}^{N-m} \sum_{i=1}^{m-1} e^{x_{k+i, i+1}-x_{k+i-1, i}} \\
\left.\left.+\sum_{k=1}^{m-1}\left[e^{-x_{N-1, k}}+\sum_{i=1}^{k-1} e^{x_{N-k+i, i+1}-x_{N-k+i-1, i}}\right]\right)\right\} .
\end{gathered}
$$


Next, let us integrate out the variables $x_{N-k, i}, k=1, \ldots, m-1, i=1, \ldots, m-k$ and $x_{m+k, m+j}, k=$ $1, \ldots, N-m-1, i=1, \ldots, k$ :

$$
\begin{gathered}
\int_{\mathbb{R} \frac{m(m-1)}{2}} \prod_{k=1}^{m-1} \prod_{i=1}^{m-k} d x_{N-k, i} \prod_{k=1}^{m-1} \exp \left\{\imath\left(\lambda_{N-k}-\lambda_{N-k+1}\right) \sum_{i=1}^{m-k} x_{N-k, i}\right. \\
-\left(\imath \lambda_{N-1-k}+\rho_{N-1-k}\right) x_{N-1-k, 1} \\
\left.-\frac{1}{\hbar}\left(e^{-x_{N-1, k}}+\sum_{i=1}^{k-1} e^{x_{N-k+i, i+1}-x_{N-k+i-1, i}}\right)\right\}=C_{m, N}^{R},
\end{gathered}
$$

and

$$
\begin{gathered}
\int_{\mathbb{R} \frac{(N-m)(N-m-1)}{2}} \prod_{k=1}^{N-m-1} \prod_{i=1}^{k} d x_{m+k, k+i} \prod_{k=1}^{N-m-1} \exp \left\{\imath\left(\lambda_{m+k}-\lambda_{m+k+1}\right) \sum_{i=1}^{k} x_{m+k, m+i}\right. \\
-\left(\imath \lambda_{m+k}-\rho_{m+k}\right) x_{m+k, m+k} \\
\left.-\frac{1}{\hbar}\left(e^{x_{N-1, m+k}}+\sum_{i=1}^{N-m-k-1} e^{x_{m+i+k-1, m+k}-x_{m+i+k, m+k}}\right)\right\}=\overline{C_{m, N}^{L}}
\end{gathered}
$$

Finally, making cancelations of the normalization constants $C_{m, N}^{R}$ and $\overline{C_{m, N}^{L}}$ in (3.2), one arrives to (1.11), and thus completes the proof of Theorem 1.1.

\section{Proof of Propositions 1.1 and 1.2}

In this part we prove Proposition 1.1. Explicit form (1.20) of the quantum Lax operator $\mathcal{L}\left(\underline{x} ; \partial_{\underline{x}}\right)$ readily follows from a simple calculation.

Lemma 4.1 The adjoint action of $g=g(\underline{x}) \in H^{(m, N)} \subset G L_{N}(\underline{1.10})$ in $\operatorname{Mat}(N, \mathbb{R})$ reads as follows:

$$
\begin{aligned}
& g^{-1} E_{11} g=E_{11}-\sum_{k=2}^{m} x_{k} E_{k, 1} \\
& g^{-1} E_{1, k} g=E_{1, k}+x_{k} E_{11}-\sum_{n=2}^{m} x_{n}\left(E_{n, k}+x_{k} E_{n, 1}\right), \quad k=2, \ldots, m \\
& g^{-1} E_{k, n} g=E_{k, n}+x_{n} E_{k, 1}, \quad k, n=2, \ldots, m \\
& g^{-1} E_{1, a} g=e^{x_{N}-x_{1}}\left[E_{1, a}+x_{a} E_{1, N}-\sum_{k=2}^{m} x_{k}\left(E_{k, a}+x_{a} E_{k, N}\right)\right], \\
& g^{-1} E_{k, a} g=e^{x_{N}-x_{1}}\left[E_{k, a}+x_{a} E_{k, N}\right], \quad k=2, \ldots, m, a=m+1, \ldots, N-1 ; \\
& g^{-1} E_{a, b} g=E_{a, b}+x_{b} E_{a, N}, \quad a=m+1, \ldots, N-1, b=a, \ldots, N-1 .
\end{aligned}
$$

Proof: Expanding the functions $F_{i j}(\underline{x})=g(\underline{x})^{-1} E_{i j} g(\underline{x})$ by the Taylor formula one arrives to (4.1). 
4.1 Next, let us introduce the following notation:

$$
\langle X g\rangle=\left\langle\psi_{L}, \pi_{\underline{\lambda}}(X) \pi_{\underline{\lambda}}(g) \psi_{R}\right\rangle, \quad X \in \mathcal{U}\left(\mathfrak{g l}_{N}\right), \quad g=g(\underline{x}) \in H^{(m, N)} .
$$

Thus, using (4.1), and taking into account the property (2.5) with the defining equations (1.6), (1.7), one can find the following:

$$
\begin{gathered}
\left\langle E_{11} g\right\rangle=\left\langle\left(E_{11}+\ldots+E_{m m}\right) g\right\rangle-\sum_{k=2}^{m}\left\langle E_{k k} g\right\rangle=\partial_{x_{1}}\langle g\rangle \\
\left\langle E_{1, k} g\right\rangle=\left\langle E_{1, k} g\right\rangle+x_{k}\left\langle E_{11} g\right\rangle+\sum_{n=2}^{m} x_{n}\left(x_{k}\left\langle E_{n, 1} g\right\rangle-\left\langle E_{n, k} g\right\rangle\right)=\left\{-\delta_{k, 2} \hbar^{-1}\right. \\
\left.+\left(1-\delta_{k, m}\right) \hbar^{-1} x_{k+1}+x_{k} \partial_{x_{1}}+\sum_{n=2}^{m} x_{k} x_{n} \partial_{x_{n}}\right\}\langle g\rangle, \quad k=2, \ldots, m ; \\
\left\langle E_{k, i} g\right\rangle=\left\{-\delta_{i, k+1} \hbar^{-1}+x_{i} \partial_{x_{k}}\right\}\langle g\rangle, \quad k=2, \ldots, m, i=k+1, \ldots, m ; \\
\left\langle E_{1, a} g\right\rangle=-(-1)^{\epsilon(m, N)} x_{a} x_{m} e^{x_{N}-x_{1}}, \\
\left\langle E_{1 N} g\right\rangle=-(-1)^{\epsilon(m, N)} x_{m} e^{x_{N}-x_{1}}, \quad\left\langle E_{m, N} g\right\rangle=(-1)^{\epsilon(m, N)} e^{x_{N}-x_{1}} ; \\
\left\langle E_{a, i} g\right\rangle=x_{i} \partial_{x_{a}}, \quad a=m+1, \ldots, N-1, \quad i=a, \ldots, N-1 ; \\
\left\langle E_{N N} g\right\rangle=\left\{\partial_{x_{N}}-\sum_{a=m+1}^{N-1} x_{a} \partial_{x_{a}}\right\}\langle g\rangle .
\end{gathered}
$$

At last, using (2.5), together with the defining equations (1.6), (1.7) one obtains the expressions for the remaining matrix elements of the Lax operator (1.20).

4.2 Finally, let us adopt the following notations:

$$
C_{I}=\sum_{\substack{i, j=1 \\ i<j}}^{N} E_{i i} E_{j j}, \quad C_{I I}=\sum_{\substack{i, j=1 \\ i<j}}^{N} E_{j i} E_{i j}, \quad C_{I I I}=\sum_{i=1}^{N} \rho_{i} E_{i i} ;
$$

and therefore $(1.17,2)$ reads

$$
C_{2}=C_{I}-C_{I I}-C_{I I I}+\sigma_{2}(\rho) \text {. }
$$

Then one has

$$
\begin{aligned}
C_{I}= & \left(E_{11}+\ldots+E_{m m}\right)\left(E_{m+1, m+1}+\ldots+E_{N N}\right)+\sum_{j=2}^{m}\left(E_{11}+\ldots+E_{m m}\right) E_{j j} \\
& +\sum_{i=m+1}^{N-1} E_{i i}\left(E_{m+1, m+1}+\ldots+E_{N N}\right)-\sum_{\substack{i, j=2 \\
i \leq j}}^{m} E_{i i} E_{j j}-\sum_{\substack{i, j=m+1 \\
i \leq j}}^{N-1} E_{i i} E_{j j}
\end{aligned}
$$

and similarly to (4.2) one finds out:

$$
\left\langle C_{I} g\right\rangle=\left\langle C_{I}^{\prime} g\right\rangle=\left\{\frac{\partial^{2}}{\partial x_{1} \partial x_{N}}+\sum_{i=m+1}^{N-1} x_{i} \frac{\partial^{2}}{\partial x_{i} \partial x_{N}}-\sum_{\substack{i, j=m+1 \\ i \leq j}}^{N-1}\left(x_{i} \frac{\partial}{\partial x_{i}}\right)\left(x_{j} \frac{\partial}{\partial x_{j}}\right)\right\}\langle g\rangle,
$$


with

$$
\begin{gathered}
C_{I}^{\prime}=\left(E_{11}+\ldots+E_{m m}\right)\left(E_{m+1, m+1}+\ldots+E_{N N}\right) \\
+\sum_{i=m+1}^{N-1} E_{i i}\left(E_{m+1, m+1}+\ldots+E_{N N}\right)-\sum_{\substack{i, j=m+1 \\
i \leq j}}^{N-1} E_{i i} E_{j j} .
\end{gathered}
$$

Next, let us observe that for $1<k \leq m$ one has

$$
\begin{gathered}
\left\langle E_{k, 1} E_{1, k} g\right\rangle=\left\{x_{k} \frac{\partial^{2}}{\partial x_{1} \partial x_{k}}-\left(-x_{k-1}\right)^{1-\delta_{k, 2}} \frac{\partial}{\partial x_{k}}\right. \\
\left.+\sum_{n=k}^{m}\left[x_{n} \frac{\partial}{\partial x_{n}}+\left(x_{k} \frac{\partial}{\partial x_{k}}\right)\left(x_{n} \frac{\partial}{\partial x_{n}}\right)\right]\right\}\langle g\rangle ;
\end{gathered}
$$

hence, for

$$
\left\langle C_{I I} g\right\rangle=\sum_{k=2}^{m}\left\langle E_{k, 1} E_{1, k} g\right\rangle+\sum_{i=m+1}^{N-1}\left\langle E_{i+1, i} E_{i, i+1}\right\rangle+\left\langle E_{m+1,1} E_{1, m+1} g\right\rangle,
$$

one obtains

$$
\begin{gathered}
\sum_{k=2}^{m}\left\langle E_{k, 1} E_{1, k} g\right\rangle=\left\{\hbar^{-1}\left(\left(\delta_{m, 1}-1\right) \frac{\partial}{\partial x_{2}}+\sum_{k=2}^{m-1} x_{k} \frac{\partial}{\partial x_{k+1}}\right)\right. \\
\left.+\sum_{k=2}^{m}\left[(k-1) x_{k} \frac{\partial}{\partial x_{k}}+\frac{\partial^{2}}{\partial x_{k}^{2}}\right]+\sum_{\substack{i, j=1 \\
i<j}}^{m}\left(x_{i}^{1-\delta_{i, 1}} \frac{\partial}{\partial x_{i}}\right)\left(x_{j} \frac{\partial}{\partial x_{j}}\right)\right\}\langle g\rangle, \\
\sum_{i=m+1}^{N-1}\left\langle E_{i+1, i} E_{i, i+1}\right\rangle=\left\{\sum_{i=m+1}^{N-2} x_{i+1} \frac{\partial}{\partial x_{i}}+\left(1-\delta_{m, N-1}\right) \hbar^{-1} \frac{\partial}{\partial x_{N-1}}\right\}\langle g\rangle, \\
\left\langle E_{m+1,1} E_{1, m+1} g\right\rangle=(-1)^{\delta_{m, N-1}+\epsilon(m, N)} \hbar^{-2}\left(x_{m}\right)^{1-\delta_{m, 1}}\left(x_{m+1}\right)^{1-\delta_{m, N-1}} e^{x_{N}-x_{1}}\langle g\rangle .
\end{gathered}
$$

At last one derives

$$
\left\langle C_{I I I} g\right\rangle=\left\{\rho_{1}\left(\frac{\partial}{\partial x_{1}}-\frac{\partial}{\partial x_{N}}\right)+\sum_{i=m+1}^{N-1}\left(\rho_{1}+\rho_{i}\right) x_{i} \frac{\partial}{\partial x_{i}}\right\}\langle g\rangle,
$$

and collecting (4.4), (4.7) and (4.8) one arrives at (1.18,2).

\section{References}

[AS] A. Astashkevich, V. Sadov, Quantum cohomology of partial flag manifolds $F_{n_{1}, \ldots, n_{k}}$, Commun. Math. Phys. 170 (1995) 503-528. [hep-th/9401103]

[BCFKvS1] V. Batyrev, I. Ciocan-Fontanine, B. Kim, D. van Straten, Conifold transitions and mirror symmetry for Calabi-Yau complete intersections in Grassmannians, Nucl. Phys. B 514 (1998) 640-666. [math.AG/9710022]

[BCFKvS2] V. Batyrev, I. Ciocan-Fontanine, B. Kim, D. van Straten, Mirror symmetry and toric degenerations of partial flag manifolds, Acta Math. 184 (2000) 1-39. [math.AG/9803108] 
[GKLO] A. Gerasimov, S. Kharchev, D. Lebedev, S. Oblezin, On a Gauss-Givental representation for quantum Toda chain wave function, Int. Math. Res. Notices, (2006) ID 96489. [math.RT/0505310].

[GLO1] A. Gerasimov, D. Lebedev, S. Oblezin, New integral representations of Whittaker fucntions for classical Lie groups, Preprint [math.RT/0705.2886], 2007, 100 pages.

[GLO2] A. Gerasimov, D. Lebedev, S. Oblezin, Parabolic Whittaker functions and Topological field theories I, Commun. Numb. Th. Phys., 5:1 (2011); [hep-th/1002.2622]

[GK] A. Givental, B. Kim Quantum cohomology of flag manifolds and Toda lattices, Commun. Math. Phys. 168, (1995), 609-641. [hep-th/9312096]

[Giv1] A. Givental, Homological geometry and mirror symmetry, Proc. ICM in Zürich, 1994, Birkhäuser, 1995, 1 472-480.

[Giv2] A. Givental, Stationary Phase Integrals, Quantum Toda Lattices, Flag Manifolds and the Mirror Conjecture. Topics in Singularity Theory, Amer. Math. Soc. Transl. Ser., 2 180, AMS, Providence, Rhode Island, 1997, 103-115. [math.AG/9612001]

[K] B. Kim, Quantum cohomology of partial flag manifolds and a residue formula for their intersection pairings, Int. Math. Res. Notices 1 (1995) 1-16. [hep-th/9405056]

[L] G. Lustzig, Total positivity in reductive groups, in Lie Theory and Geometry: In Honor of B. Kostant, Progr. Math. 123, Birkhäuser, 1994, 531-568.

[O] S. Oblezin, On parabolic Whittaker functions, Preprint [math.AG/1011.4250], 2010, 15 pages.

Institute for Theoretical and Experimental Physics, Bol. Cheremushininsaya 25, Moscow 117218,

E-mail address: Sergey.Oblezin@itep.ru 